\title{
Topical microemulsion containing Punica granatum extract: its control over skin erythema and melanin in healthy Asian subjects
}

\author{
Rashida Parveen ${ }^{1}$, Naveed Akhtar ${ }^{1}$, Tariq Mahmood ${ }^{2}$
}

1Department of Pharmacy, Faculty of Pharmacy and Alternative Medicine, The Islamia University of Bahawalpur, Bahawalpur, Pakistan ${ }^{2}$ Faculty of Pharmacy, University of Central Punjab, Lahore, Pakistan

Postep Derm Alergol 2014; XXXI, 6: 351-355

DOI: 10.5114/pdia.2014.47117

\begin{abstract}
Introduction: Punica granatum is apotent source of polyphenolic compounds with strong free radicals scavenging activity. The skin lightening effects of Punica granatum are assumed due to ellagic acid which acts by chelating copper at the active site of tyrosinase.

Aim: To explore a topical microemulsion (O/W) of pomegranate (Punica granatum) extract for its control on skin erythema and melanin.

Material and methods: Microemulsions were formulated using a polysorbate surfactant $\left(\right.$ Tween $\left.80^{\circledR}\right)$ along with cosurfactant (propylene glycol) and were characterized regarding their stability. The placebo microemulsion (without extract) and the active microemulsion (containing Punica extract) were applied in a split face fashion by the volunteers $(n=11)$ for a period of 12 weeks. Skin erythema and melanin were measured at baseline and after every 15 days to determine any effect produced by these formulations.

Results: Active formulation showed a significant impact on skin erythema and melanin $(p<0.05)$.

Conclusions: This study reveals that a suitable topical formulation like microemulsion could employ the Punica granatum extract for conditions where elevated skin melanin and erythema have significantly prone skin physiology.
\end{abstract}

Key words: Punica granatum extract, antioxidant, microemulsion, melanin, erythema.

\section{Introduction}

Skincolor has become an important phenotypic characteristic for which many natural and synthetic chemical agents are used to modulate the metabolism of the pigmentation produced [1]. Fascination with novel topical formulations loaded with functional actives having better antioxidant activity have emerged in the recent era [2]. There is an increasing interest in evaluating antioxidants for their shielding effects against a variety of degenerative diseases [3]. In Ayurveda medicine, Punica granatum is considered "A Pharmacy unto Itself" [4]. This herb was used in ancient Egypt for treatment of inflammation of the skin, mucosa and joints [5]. Pomegranate is the potent source of polyphenolic compounds, i.e., flavonoids and hydrolysable tannins (punicalin, pedunculagin, punicalagin, gallic acid and ellagic acid) which have shown up to $92 \%$ radical scavenging activity $[6,7]$. The skin lighten- ing effects of ellagic acid are thought to be due to chelating copper at the active site of tyrosinase. Ellagic acid has skin whitening, moisturizing, sun protecting, anti-wrinkle, anti-acne, anti-stain and anti-freckles effects [8, 9].

More recently, a cream containing a phenolic-rich extract of Acacia bark has been tested for its efficacy on the human skin against its melanin and erythema effects [10]. A microemulsion, made from water, oil, surfactants and cosurfactant is a thermodynamically stable system [11]. Microemulsions are fluid and isotropic formulations that have been widely studied as delivery systems for a variety of routes, including the skin. In spite of what the name suggests, microemulsions are nanocarriers, and their use as topical delivery systems derives from their multiple advantages compared to other dermatological formulations, such as ease of preparation, thermodynamic stability and penetration-enhancing properties [12]. More recently, Gly-

Address for correspondence: Tariq Mahmood PharmD, MPhil, PhD, RPh, Faculty of Pharmacy, University of Central Punjab, 1 - Khayaban-e-Jinnah Road, Johar Town, 54000 Lahore, Pakistan, phone: 0923332322 200, e-mail: dr.tariq@ucp.edu.pk Received: 6.05.2014, accepted: 10.09.2014. 
cyrrhiza glabra root and rhizome aqueous ethanolic extract in microemulsion carrier systems has been formulated for transdermal delivery of incorporated antioxidant actives, flavonoids and polyphenols [13].

\section{Aim}

This study was aimed to depict efficacy of Punica granatum extract contained in a microemulsion formulation against skin melanin and erythema of healthy Asian subjects.

\section{Material and methods}

A measuring probe, Mexameter ${ }^{\circledR}$ MX 18 (Courage Khazaka, Germany) was used to take measurements of skin melanin and erythema. Tween $80^{\circledR}$ (Franken Chemical, Germany) was used as hydrophilic emulsifier. Propylene Glycol (Merck, Germany) was used as co-surfactant. Palm oil was used as oil phase. Other instruments used were Electrical Balance (Precisa BJ-210, Switzerland) and Hot plate Magnetic stirrer (Velp Scienifica, Germany). Distilled water obtained through the Department of Pharmacy, the Islamia University of Bahawalpur, Pakistan. Shade-dried ground Punica granatum fruit rinds (Peels) were used as plant material.

\section{Preparation of microemulsions}

Microemulsions were prepared by the spontaneous emulsification technique (phase titration method) which is easy to depict with the help of a phase diagram. Different ratios of the surfactant (Tween $80^{\circledR}$ ) and cosurfactant (propylene glycol) were used in the preparation of the microemulsion. For construction of the phase diagram, oil, surfactant mixture and water were weighed and mixed together at room temperature. After each mixing, the sample was allowed to equilibrate for $24 \mathrm{~h}$. The microemulsion regions in the pseudoternary phase diagram were determined.

\section{Characterization of microemulsions}

\section{Particle size determination}

The size of globule was measured at $25^{\circ} \mathrm{C}$ by a Zetasizer (Malvern Instruments Ltd., ZEN3600, UK). Samples were placed in clear disposable zeta cells and results were recorded.

\section{Conductivity measurements}

Electrical conductivity of MEs was measured with a conductivity meter (Conductometer WTW - Cond 197i, Germany). All measurements were carried out in triplicate.

\section{Determination of $\mathrm{pH}$}

The $\mathrm{pH}$ values for microemulsion were determined at $25^{\circ} \mathrm{C}$ by pH meter (WTW pH-197i, Germany). All measurements were carried out in triplicate.

\section{Tested formulations}

The tested formulations in this study were O/W microemulsions (placebo and active formulation). The microemulsions were named $A$ (active formulation containing the extract) and B (placebo with no extract).

\section{Assessment of skin irritation}

To assess any skin irritation in selected subjects, a patch test was performed on both forearms of each volunteer before the start of the efficacy study. Small regions were marked on the forearms. The patch (Bandage disc) for the left forearm was saturated with a small amount of the placebo formulation while the patch on the right forearm was saturated with a small amount of active formulation. Each patch was applied to the marked regions separately on each forearm. The regions were covered with the surgical dressing after application. The patches were removed after $48 \mathrm{~h}$ and the forearms were observed for any skin irritation by the investigator.

\section{Product evaluation on skin}

A total of 11 male volunteers aged $25-40$ years were selected for the study after they have signed a consent form. The volunteers were examined by an expert investigator for any skin damage before the start of the efficacy study. The study was designed as single-blinded, placebo control to establish comparisons of two microemulsion formulations. The experiments were carried out on the cheeks of volunteers as cheeks are uniformly and more prone to ultraviolet (UV) radiations. Every volunteer applied the microemulsion at night on cheeks for a period of 12 weeks and returned for the measurements on the $2^{\text {nd }}, 4^{\text {th }}, 6^{\text {th }}, 8^{\text {th }}, 10^{\text {th }}$ and $12^{\text {th }}$ week. They were allowed for routine face wash. Before every measurement volunteers were acclimatized with room conditions for at least $30 \mathrm{~min}$. All measurements were performed by a single expert investigator to minimize person to person variation and room conditions were maintained as per standard protocols.

\section{Ethical standards}

This study was approved by the Board of Advanced Studies and Research (BASR), and its Ethical Committee to conduct efficacy studies (Reference No 3715/Acad), The Islamia University of Bahawalpur. All the protocols were taken into the consideration according to international guidelines of Helsinki Declaration.

\section{Calculation of percent changes}

The percentage changes for the individual values of skin erythema and melanin of volunteers were calculated by the following formula: percentage change $=[(D x-D 0) / D 0] \times$ 100 (1), where $D x$ is the individual value of any parameter on the $2^{\text {th }}, 4^{\text {th }}, 6^{\text {th }}, 8^{\text {th }}, 10^{\text {th }}$ or $12^{\text {th }}$ week and $D O$ is the $0 \mathrm{~h}$ value of that parameter. 


\section{Statistical analysis}

Paired samples $t$-test for variation between the two preparations and one-way ANOVA for variation between different times intervals were applied using SPSS 12.0 on computer using a $5 \%$ level of significance.

\section{Results and discussion}

Pseudo-ternary phase diagrams of the investigated microemulsion with different surfactant-cosurfactant ratios are shown in Figure 1. Microemulsions were formed at ambient temperature. The phase diagrams clearly indicated the microemulsion existence region in the weight ratio of surfactant/cosurfactant $\left(S_{\text {mix }}=5: 1\right)$.

Particle sizes of placebo microemulsion and microemulsion containing the Punica granatum extract were determined and there were no significant differences in average particle size after loading the drug. The microemulsion containing the pomegranate extract showed the average particle size of $8.08 \pm 0.2 \mu \mathrm{m}$ with polydispersity index (PI) of $0.362 \pm 0.034$ (Table 1). Polydispersity index is a measure of particle homogeneity and it varies from 0.0 to 1.0. The closer to zero the PI value the more homogenous are the particles [14]. There was a strong correlation between the specific structure of the microemulsion systems and their electrical conductivity behavior. Formation and type of the microemulsion formed was also confirmed with a color test.

The blank microemulsion and microemulsion containing the Punica granatum extract showed a skin suitable $\mathrm{pH}$ value (5.97 to 5.65, respectively). The decrease in $\mathrm{pH}$

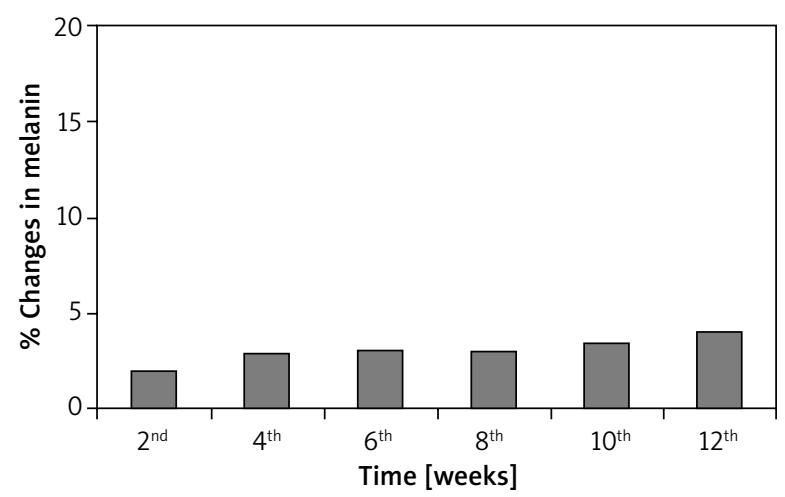

Figure 2. Percentage of changes in skin melanin after application of placebo

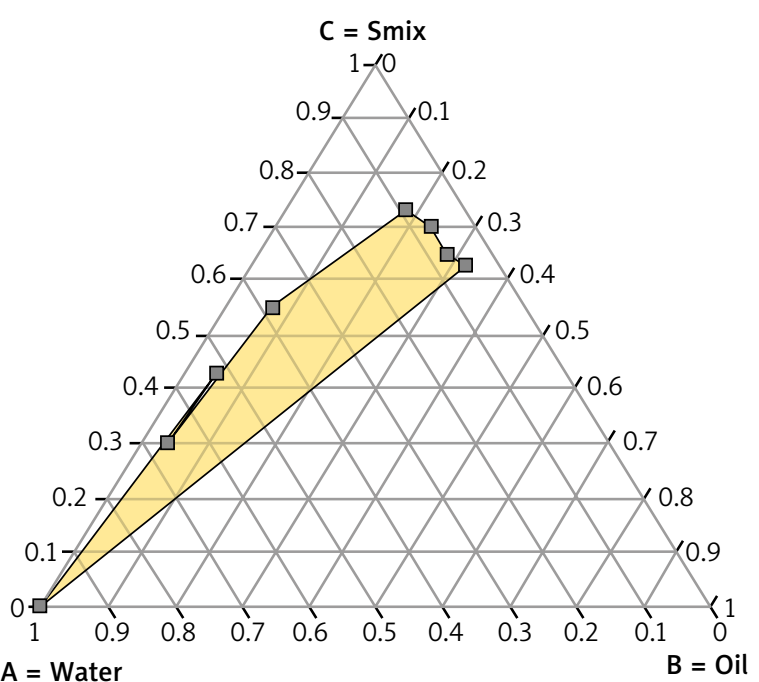

Figure 1. Pseudo ternary phase diagram of surfactant mixture, oil and water $\left(\mathrm{S}_{\text {mix }} 5: 1\right)$

of the formulation at storage conditions might be due to the production of any acidic metabolite that best suits for a topical application. Incorporation of the pomegranate extract has no effect on the observed $\mathrm{pH}$ values of the microemulsions (Table 1).

In efficacy studies, it was found that there was an increase in skin melanin values after the application of a placebo microemulsion and on the other side, active formulation continuously decreased skin melanin contents throughout the observation period (Figures 2 and 3).

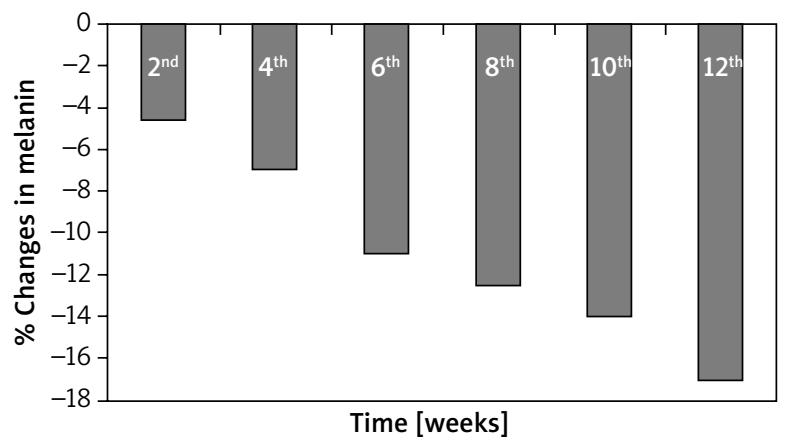

Figure 3. Percentage of changes in skin melanin after application of active formulation

Table 1. Characteristics of placebo microemulsion and the Punica granatum extract containing microemulsion $(n=3$ with SD)

\begin{tabular}{lccc}
\hline Description & Particle size $[\mu \mathrm{m}]$ & Conductivity $[\mu \mathrm{S} / \mathrm{cm}]$ & $\mathrm{pH}$ \\
\hline Blank microemulsion & $9.28 \pm 0.4$ & $7.1 \pm 0.3$ & $5.97 \pm 0.5$ \\
\hline Microemulsion containing extract & $8.08 \pm 0.2$ & $6.3 \pm 0.1$ & $5.65 \pm 0.3$ \\
\hline
\end{tabular}




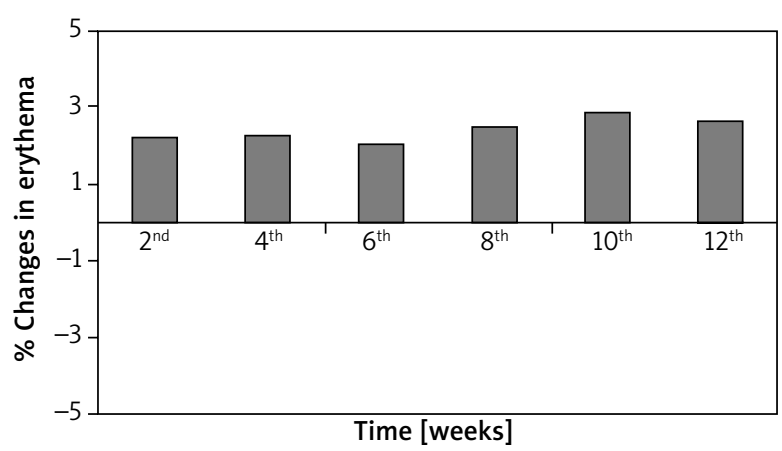

Figure 4. Percentage of changes in skin erythema after application of placebo

With the help of ANOVA test, it was found that changes in skin melanin values after the application of active formulation were significant $(p<0.05)$ with respect to time. Furthermore, a paired sample $t$-test indicated that active formulation produced more significant $(p<0.05)$ effects at the $6^{\text {th }}, 8^{\text {th }}, 10^{\text {th }}$ and $12^{\text {th }}$ week incomparison to placebo formulation. The decrease in skin melanin content after application of formulation may be attributed to the antioxidant activity of the pomegranate extract which is rich in ellagic acid, punicalagin and vitamins C [15]. Ellagic acid causes inhibition of tyrosinase and melanin synthesis thus inhibiting melanogenesis [16].

For the safety of cosmetics, the important point is that cosmetics must not cause any contact dermatitis when applied to the skin. Skin irritation is caused by the direct toxicity of chemicals on cells or blood vessels in the skin and is different from contact allergy which is caused by immune response [17]. In the present study, placebo formulation produced no effects in reducing skin erythema at different time intervals, whereas application of active formulation produced significant $(p<0.05)$ effects on skin erythema at different time intervals. With the help of paired sample $t$-test it was evident that there was no significant variation in irritation potential when placebo and active formulations were compared. Effects on erythema appear due to antioxidant and anti-inflammatory constituents of the Punica granatum extract [18] (Figures 4 and 5).

\section{Conclusions}

It has been concluded that a stable microemulsion formulation of the Punica granatum extract can be formulated with considerable stability and skin compatibility characteristics. Results of the efficacy study in Asian healthy subjects suggest that Punica granatum has significant control over skin erythema and pigmentation. Further studies in patients with pigmentation disorders could warrant the actual potential of Punica granatum in unhealthy states.

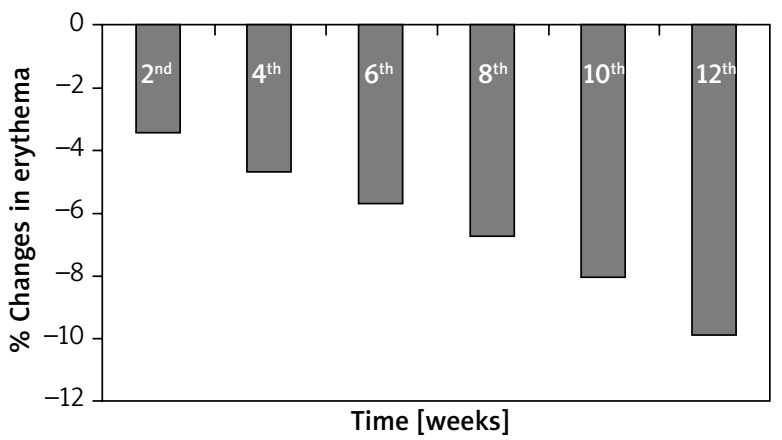

Figure 5. Percentage of changes in skin erythema after application of active formulation

\section{Acknowledgments}

The authors are highly obliged for the financial support by the Higher Education Commission of Pakistan.

\section{References}

1. Stratigos AJ, Katsambas AD. Optimal management of recalcitrant disorders of hyperpigmentation in dark-skinned patients. Am J Clin Dermatol 2004; 5: 161-8.

2. Mahmood T, Akhtar N, Manickam S, et al. Interfacial film stabilized W/O/W nano multiple emulsions loaded with green tea and lotus extracts: systematic characterization of physicochemical properties and shelf-storage stability. J Nanobiotechnol 2014; 12: 20.

3. Yigit D, Yigit N, Mavi A, et al. Antioxidant and antimicrobial activities of bitter and sweet apricot (Prunus armeniaca L.) kernels. Braz J Med Biol Res 2009; 42: 346-52.

4. Ephraim PL, Robert AN. Punica granatum (Pomegranate) and its potential for prevention and treatment of inflammation and cancer. J Ethnopharmacol 2007; 109: 177-206.

5. Carl TT. Cosmeceuticals containing herbs: fact, fiction, and future. Dermatol Surg 2005; 31: 873-80.

6. Deeba NS, Farrukh A, Hasan M, et al. Pomegranate derived products for cancer chemoprevention. Sem Can Biol 2007; 17: 377-85.

7. Maria IG, Francisco A, Tomas B, et al. Antioxidant activity of pomegranate juice and its relationship with phenolic composition and processing. J Agric Food Chem 2000; 48: 4581-9.

8. Mineka Y, Yuko W, Kouichi K, et al. Effect of an ellagic acid rich pomegrante extract on tyrosinase activiy and ultra-violet induced pigmenation. Biosci Biotechnol Biochem 2008; 12: 2368-73.

9. Sharma SR, Poddar R, Sen P, et al. Effect of vitamin C on collagen biosynthesis and degree of birefringence in polarization sensitive optical coherence tomography (PS-OCT). Afr J Biotechnol 2008; 7: 2049-54.

10. Ali A, Akhtar N, Khan MS, et al. In vivo evaluation: the effects of a cream containing Acacia bark extract on skin melanin and erythema content. Postep Derm Alergol 2012; 29: 369-72.

11. Karasulu HY. Microemulsions as novel drug carriers: the formation, stability, applications and toxicity. Expert Opin Drug Deliv 2008; 5: 119-35.

12. Lopes LB. Overcoming the cutaneous barrier with microemulsions. Pharmaceutics 2014; 6: 52-77.

13. Mostafa DM, Ammar NM, Abd El-Alim SH, et al. Transdermal microemulsions of Glycyrrhizaglabra L.: characterization, 
stability and evaluation of antioxidant potential. Drug Deliv 2014; 21: 130-9.

14. Kashyap N, Jadon P, Naruka P, et al. Formulation development and characterization of aceclofenac gel using poloxamer 407. J Chem Pharm Res 2010; 2: 357-63.

15. Kulkarni AP, Aradhya SM. Chemical changes and antioxidant activity in pomegranate arils during fruit development. Food Chem 2005; 93: 319-24.

16. Kasai K, Yoshimura M, Koga T, et al. Effect of oral administration of ellagic acid rich pomegranate extract on ultra-violet induced pigmentation in the human skin. J Nutr Sci Vitaminol 2006; 52: 383-8.

17. Muhammad NA, Ephraim PL, James V, et al. Pomegranate as a cosmeceutical source: pomegranate fractions promote proliferation and procollagen synthesis and inhibit matrix metalloproteinase-1 production in human skin cells. J Ethnopharmacol 2006; 103: 311-8.

18. Jean PO, Donald LB. Latest insights into skin hyper pigmentation. J Invest Dermatol 2008; 13: 10-4. 